\title{
TREATMENT OPTIMIZATION OF PATIENTS WITH GENITAL ENDOMETRIOSIS
}

\author{
Nikolay Shcherbina \\ Department of Obstetrics and Gynecology No. $1^{1}$ \\ Anastasiia Chekhunova \\ Department of Obstetrics and Gynecology No. $1^{1}$ \\ chekhunova1606@gmail.com \\ ${ }^{1}$ Kharkiv National Medical University \\ 4 Nauky ave., Kharkiv, Ukraine, 61022
}

\begin{abstract}
The aim of the research: to optimize the treatment of patients with combined genital pathology, including internal endometriosis (adenomyosis) and inflammatory diseases.

Materials and methods: prospective study has been conducted on 160 women with adenomyosis. There were 24 (15\%) patients with the I degree of adenomyosis spreading, 72 (45.0\%) women with the II degree, 33 (20.6\%) patients with the III degree, and $31(19.4 \%)$ woman with the IV degree of adenomyosis spreading. Microbial flora analysis included bacterioscopic, bacteriological research methods with determination of sensitivity to antibiotics, and PCR method.

The concentration of cytokines in the culture medium (supernatant) was determined by the enzyme immunoassay.

Results: the obtained data from the study indicate a high percentage of the combination of adenomyosis with chronic inflammatory diseases of the pelvic organs. An immuno-inflammatory reaction preceding adenomyosis is accompanied by the violation of the local cytokine balance. In turn, the increased activity of cytokines and the presence of infectious agents can participate in the relapse of endometriosis.

Conclusion: considering the immuno-inflammatory reaction, accompanied by the violation of the local cytokine balance in the development of adenomyosis. The study substantiates the necessity of using antimicrobial therapy in patients with combined genital pathology, including adenomyosis and inflammatory diseases.

Keywords. Adenomyosis, genital chronic inflammatory diseases, cytokine imbalance, antimicrobial therapy, relapse of the disease.
\end{abstract}

DOI: $10.21303 / 2504-5679.2021 .001682$

\section{Introduction}

The relevance of preserving and restoring the reproductive health of women is of particular importance in connection with the increase in the number of chronic inflammatory diseases of the pelvic organs and genital endometriosis in modern women, with a lack of knowledge of the pathogenetic mechanisms of the occurrence of combined pathology, with the peculiarities of the clinical course, with the difficulties of early diagnosis and the ineffectiveness of traditional methods of treatment [1]. Chronic inflammatory diseases of female genital organs, occupying a leading place in the structure of gynecological pathology, entail disturbances in the nervous, endocrine and immune systems of the body [2,3]. Genital endometriosis ranks third in the structure of gynecological pathology after inflammatory diseases [4]. Analysis of the literature on the state of the non-specific, humoral and cellular links of the immune system in combined pathology of the genitals indicates the inconsistency of the results obtained by the author [5, 6]. So, for example, genital endometriosis, which is a polyetiological and polypathological process, is characterized by the inclusion of an inflammatory reaction in the focus of endometriosis [7, 8]. In addition, many clinical manifestations of endometriosis either arise against the background of previous inflammatory processes, or cause the manifestation of «inflammatory factors» in the endometrioid focus $[9,10]$.

One of the areas of treatment for both endometriosis and chronic salpingo-oophoritis is the pharmacotherapy $[11,12]$. The purpose of drug therapy is to stop the process development [13]. However, the multicenter studies showed that the effectiveness of this therapy was $67-70 \%$ and the number of relapses of endometriosis a year after drug withdrawal reached 12-37\% [14]. The endo- 
metriosis recurrence rate increased in case of its combination with chronic inflammatory diseases of the genitals, which was evidenced by the high infectious index (97.2\%) in such patients [15].

Thus, development of individual approaches to examination and selection of optimal treatment tactics, taking into account the patient's age, localization of the endometrioid process, degree of distribution, combination with genital pathology, is an urgent problem of gynecology $[16,17]$.

The aim of this article was to optimize the treatment of patients with combined genital pathology, including internal endometriosis (adenomyosis) and inflammatory diseases.

\section{Material and methods}

All experiments were conducted between 2019-2021 at the clinical bases of the Department of Obstetrics and Gynecology No. 1, Kharkiv National Medical University, Kharkiv, Ukraine.

Prospective study has been conducted on 160 patients with adenomyosis. There were 24 (15\%) patients with the I degree of adenomyosis spreading, 72 (45.0\%) women with the II degree, $33(20.6 \%)$ patients with the III degree, and 31 (19.4\%) woman with the IV degree of adenomyosis spreading. The average age of the examined was $27.3 \pm 1.6$ years. Patients were divided into three groups depending on the type of the used hormone therapy. Group I included $52(32.5 \%)$ patients who received Janine. 57 (35.6\%) of patients in group II were administered Gestrinone for 6 months. $51(31.9 \%)$ patients in group III received the drug Zoladex for 6 months. Relapses after treatment were recorded in $58(36.3 \%)$ patients. The control group consisted of 30 healthy fertile women.

Ethical approval was obtained from the Bioethics committee of Kharkiv National Medical University (protocol No. 2, 10.06.2020). All participants consented to take part in the study. All experiments involving participants were conducted in accordance with World Medical Association Declaration of Helsinki.

Experimental procedures. Comprehensive examination included the study of the nature of complaints, anamnesis, transvaginal ultrasound, colposcopic, and hysteroscopic studies. Studies of endometrial tissue and epithelium of the cervical canal and vagina to determine the microbial flora included bacterioscopic, bacteriological research methods with determination of sensitivity to antibiotics, and PCR method.

The concentration of cytokines in the culture medium (supernatant) was determined by the enzyme immunoassay using commercial test systems («Protein circuit») [18].

Statistical processing of data was performed using the Med Stat program according to the recommendations of S. Glanz (1999) [19]. When comparing groups, the Mann-Whitney test was used, the differences were considered significant at $p<0.05$.

\section{Research results}

Studying menstrual function, we found out that 51 (32\%) patients established their menstrual cycle in the period from 6 months to 2 years, which indicated the functional inferiority of various parts of the hypothalamus-pituitary-ovary system. Algodismenorrhea occurred with menarche $(p<0.05)$ in every second patient. $31.3(66.7 \%)$ of the examined patients had artificial abortions in their medical records. 35 (21.9\%) of the examined patients suffered from primary infertility, and $31(19.3 \%)$ had secondary infertility. $16(10 \%)$ of all the examined patients had physiological childbirth in their medical records. 48 (30\%) of cases had various surgical interventions during childbirth.

When studying extragenital pathology, we found that patients with adenomyosis frequently had acute respiratory infections (83.8 \%), the frequency of childhood infections was $79.4 \%$. Noteworthy is the high incidence of gastrointestinal diseases (gastritis, colitis, sigmoiditis), which was $33.1 \%$.

Thus, we observed various somatic pathologies in most patients, often combined with foci of local infection, which may be explained by disturbances of the immune system in these patients.

In the structure of gynecological diseases, $95.6 \%$ of patients had inflammatory diseases of the female genital organs. Their structure and frequency were as follows: colpitis was diagnosed in 105 (68.6\%) patients; chronic salpingo-ophoritis was observed in 65 (42.5\%) patients; 55 (36\%) had chronic endometritis; and 107 (70\%) had endocervicitis.

Coming to treatment, 120 (75\%) women complained about painful menstruation, 74 (46.3\%) had pelvic pain, and 94 (58.8\%) complained about hyperpolymenorrhea. The pain 
index (PI) was 7.2 \pm 0.6 . Gynecological examination revealed a slight increase in the uterus, corresponding to a 5-6-week pregnancy in $83(51.8 \%)$ patients, the uterus was enlarged for more than 5-6 weeks of pregnancy (combination with myoma) in 77 (48.1\%) patients.

On examination in the mirrors and colposcopy of the vaginal mucosa and cervix, cervical ectropion was detected in 39 (24.4\%) patients.

During hysteroscopy, we fixed the following main hysteroscopic signs of internal endometriosis: uneven contours of the uterine cavity, a rocky, looped wall pattern, and the presence of crypts. Hysteroscopy was combined with targeted biopsy and subsequent histological examination of the biopsy. A histological examination of biopsy samples in 75 (46.9 \%) patients with adenomyosis revealed an endometrium of the secretory type. The glandular cystic endometrial hyperplasia was noted in $21(13.1 \%)$. The obtained material also testified to the presence of inflammatory process in the endometrium and the contents of the cervical canal, which were revealed by histological examination in 133 (83\%) patients. Thus, according to our study, inflammatory changes in the endometrium and epithelium of the cervical canal were combined with adenomyosis in $83 \%$ of cases.

The high frequency of concomitant inflammatory pathology occurrence in the genitals allowed us to conduct an extended study of the microflora in patients with adenomyosis.

Representatives of the Mollicutes class (M hominis, Genitalium, U urealyticum/parvum) occupied the dominant place in the cervical canal epithelium and were detected in 78 (58.6\%) women. Chlamidia trachomatis and Herpes simplex virus I, II were found in the epithelium of the cervical canal in 19 (14.3\%) patients. Bacterial vaginosis was diagnosed in 49 (36.8 \%) patients, Candida albicans (vaginal candidiasis) was found in 33 (24.8\%) patients. A study of the structure of the association of vagina microbial flora and cervical canal showed that monoinfection was detected in 34 (25.6\%) patients, and associations of pathogens from two or more species were found in 98 (74.7\%) patients $(p<0.05)$.

The analysis of the endometrium microflora study results showed a significant predominance $(p<0.005)$ of representatives of the Mollicutes class (M. hominis, M. genitalium, U. urealyticum/parvum). They were found in $56(42.1 \%)$ of the examined women. In the structure of the microbial flora obtained from the endometrium, Gardnerella vaginalis was detected in $15(11.3 \%)$ patients, Candida albicans was in $8(6 \%)$ examined women, Chlamidia trachomatis in the endometrium was found in $12(9 \%)$ patients, Trichomonas vaginalis was detected in $8(6 \%)$ patients, Herpes simplex virus I, II was diagnosed in the endometrium of 10 (7.5\%) patients, pathogenic streptococcus was detected in the endometrium of $8(6 \%)$ women, pathogenic staphylococcus was diagnosed in $4(3 \%)$ patients.

After revealing a pathogenic genital infection, all patients underwent etiotropic antibacterial and antiviral therapy prior to hormone therapy.

After the treatment, the number of complaints decreased. The frequency of dysmenorrhea significantly decreased by $45 \%$, pelvic pain disappeared in $54 \%$ of patients. A positive effect was observed in the characteristic of menstruation (the number of discharge and days of menstruation decreased). PI decreased during treatment and amounted to $3.1 \pm 0.02$ points, which corresponded to a mild degree of pain syndrome.

During treatment, patients with adenomyosis showed a decrease in the endometrium thickness compared with the endometrium thickness before treatment $(p<0.05)$. During control color Doppler mapping in patients with adenomyosis after treatment, minimal vascularization was recorded in $92 \%$ of cases after treatment (the exception was patients with adenomyosis of III-IV stage). The resistance index in patients of I and II degree of adenomyosis was $0.48 \pm 0.03$ (before treatment $0.76 \pm 0.03$ ), this indicator allows us to assess the adequacy of therapy, and probably to predict the absence of relapse after treatment. After the treatment, we observed a decrease in CA 125 concentration (before treatment it was $19.7 \mathrm{U} / \mathrm{ml}$, after treatment $-12.4 \mathrm{U} / \mathrm{ml}$ ) in these patients. The decrease in the dynamics of the tumor marker CA 125 by 1.6 times is also a diagnostic criterion for the effectiveness of therapy, a prognostically important criterion for the course of the disease.

At the present stage, the effectiveness of the treatment of adenomyosis is assessed by the following criteria: the state of reproductive function (pregnancy rate), the severity of clinical symptoms, the frequency of relapses, and the normalization of the menstrual cycle. The number of pregnancies after a year treatment was $11.5 \%$ of pregnancies in patients of group I; $12.3 \%$ of pregnancies in group II women, and $25.5 \%$ of pregnancies in patients of group III. The clinical symptoms 
of adenomyosis decreased or completely disappeared during therapy in $46(88.5 \%)$ patients of group I, in $48(84.2 \%)$ patients of group II, and in $49(96.1 \%)$ women of group III $(p>0.05)$.

The number of women with heavy menstruation after treatment, decreased in group I by $57.7 \%$, in group II it decreased by $52.6 \%$, in group III it decreased by $45.1 \%$. The pain during menstruation decreased by $53.8 \%$ in group I patients, by $45.6 \%$ in group II patients, and by $45.1 \%$ in group III patients after treatment.

It should be noted that relapses of adenomyosis during the year after the appointment of antimicrobial therapy were observed in 18 (13.5\%) of the examined. Relapses were observed in $7(13.5 \%)$ patients of group I, in $8(14 \%)$ and $7(13.7 \%)$ patients of comparison groups, respectively. We conducted a re-examination in case of relapse in patients after the treatment to detect microbial factors in the endometrium, epithelium of the cervical canal and vagina.

As a result of the examination, Herpes simplex virus I, II virus was diagnosed in the cervical canal of $15(83.3 \%)$ patients and in the endometrium of $10(55.6 \%)$ patients. Bacterial vaginosis was diagnosed in the vagina of $13(72.2 \%)$ patients, Gardnerella vaginalis was detected in the endometrium of $9(50.0 \%)$ patients. Etiotropic treatment was repeated for these patients.

One of the reasons for the frequent recurrence of the disease is the failure of the body protective systems and a change in intercellular interactions. When studying the cytokine profile of cervical contents, we detected an increase in the concentration of the main pro-inflammatory cytokines IL-1 $\beta$, IL-6, TNF $\alpha$ and a factor with the chemotoxic properties of IL-8. To the greatest extent, we observed an increase in the concentration of IL-1 $\beta$, TNF $\alpha$ among the studied cytokines (Table 1).

Table 1

The content of cytokines in cervical contents of patients with adenomyosis

\begin{tabular}{lccc}
\hline \multirow{2}{*}{ Cytokines pg/ml } & \multicolumn{2}{c}{ Patients with adenomyosis } & \multirow{2}{*}{ Healthy fertile women $(\boldsymbol{n}=\mathbf{3 0})$} \\
\cline { 2 - 4 } & I-II degree $(\boldsymbol{n}=\mathbf{9 6})$ & III-IV degree $(\boldsymbol{n}=\mathbf{6 4})$ & $2.2 \pm 1.5$ \\
\hline \multirow{2}{*}{ IL-1 $\beta$ spontaneous induced } & $12.5 \pm 3.4^{*}$ & $17.2 \pm 2.4^{*}$ & $32.7 \pm 3.1$ \\
& $31.3 \pm 3.2$ & $37.2 \pm 3.7$ & $8.3 \pm 1.9$ \\
IL-6 spontaneous induced & $9.7 \pm 2.1$ & $11.4 \pm 4.6^{*}$ & $24.5 \pm 2.8$ \\
& $25.6 \pm 6.2$ & $31.4 \pm 6.5$ & $1.2 \pm 0.3$ \\
TNF- $\alpha$ spontaneous induced & $2.1 \pm 0.4^{*}$ & $1.92 \pm 3.4^{*}$ & $26.7 \pm 2.5$ \\
& $25.9 \pm 1.8$ & $32.5 \pm 3.7$ & $4.3 \pm 0.6$ \\
IL-8 spontaneous induced & $7.9 \pm 0.9^{*}$ & $13.4 \pm 4.9^{*}$ & $36.7 \pm 3.6$
\end{tabular}

Note: ${ }^{*}-p<0.05$ is significance of differences in relation to healthy people

Local cytokine imbalance can be either a cause of a decrease in the level of immune response aimed at restoring local homeostasis and maintaining the normal functioning of organs, or altering immune responses, giving them an aggressive character. The increase in the content of IL-8 in patients with adenomyosis indicates the involvement of their immune system in the pathogenesis of this disease as an inflammatory reaction and at the same time reflects the participation of this interleukin in the proliferative activity of endometrioid heterotopia.

\section{Discussion}

In recent decades, a number of studies have been published that characterize the development of endometriosis from the viewpoint of immunological disorders [20, 21]. Deserves special attention the idea of [22] bacterial contamination as a factor of the development of endometriosis, which was proposed in 2008 by Kodati V. L. [23]. In 2018, Khan K. N. and co-authors proposed the concept of an increase in the role of bacterial contamination in patients with endometriosis against the background of a decrease in the expression of antimicrobial peptides in the epithelium during menstruation, which can further lead to invasion of endometrioid heterotopies [24]; Apparently, microbial associations found in the female reproductive tract can influence immune responses. The nature of the interaction between microbial associations and immune cells is unknown. In work [25] on mouse 
models with endometriosis, treatment with broad-spectrum antibiotics was carried out, which led to a decrease in endometrioid lesions, confirming the possible role of microbes in the progression of endometriosis. The expediency of detailing the pathogenetic aspects of adenomyosis is due to the improvement of diagnosis and the inclusion in therapy of measures related to changes in the function of various components of the immune system and the involvement of the infectious «beginning».

Publications of recent years indicate a significant increase in the prevalence of adenomyosis, accompanied by an increase in the frequency of surgical interventions on the organs of the reproductive system, demonstrating the social aspect of the problem.

The analysis of scientific reviews convinces of the low effectiveness of therapeutic and prophylactic measures at the present stage, explaining this fact by the absence of their etiological orientation due to insufficient knowledge about the key mechanisms of proliferative processes in the uterus with adenomyosis.

Study limitations. The study of homeostatic relationships of microbial associations and local immune mechanisms, analysis of the potential effects of anti-inflammatory therapy can be obtained in an experiment on animals, which is possible in the future research.

A prospective study of the pathogenetic aspects of the development of adenomyosis will allow not only to determine new diagnostic aspects, but also to provide effective approaches to the differentiated therapy of this pathology.

\section{Conclusions}

Thus, cytokines (their increased activity and imbalance) can act as factors potentiating proliferative activity in the development of adenomyosis, and thereby participate in the recurrence of the endometrioid process. The presence of infectious agents can likely disrupt the receptor apparatus of the uterus, ultimately leading to a relapse of the disease. The obtained data indicate that the development of adenomyosis requires a previous immuno-inflammatory reaction, accompanied by the violation of the local cytokine balance.

The obtained data showed a high percentage of the combination of adenomyosis with chronic inflammatory diseases of the pelvic organs and substantiated the need for antimicrobial therapy in this category of patients. The positive dynamics in the treatment of patients with adenomyosis in $86.5 \%(p<0.05)$ of cases, and a decrease in the frequency of adenomyosis relapses by 2.4 times confirm the study results. That is why we can state that we have developed and proposed an algorithm for the optimal treatment of patients with combined genital pathology, including adenomyosis and inflammatory diseases.

\section{Conflict of interests}

The authors declare that they have no conflicts of interest.

\section{References}

[1] Lin, W.-C., Chang, C. Y.-Y., Hsu, Y.-A., Chiang, J.-H., Wan, L. (2016). Increased Risk of Endometriosis in Patients With Lower Genital Tract Infection. Medicine, 95 (10), e2773. doi: http://doi.org/10.1097/md.0000000000002773

[2] Bukulmez, O., Hardy, D. B., Carr, B. R., Word, R. A., Mendelson, C. R. (2007). Inflammatory Status Influences Aromatase and Steroid Receptor Expression in Endometriosis. Endocrinology, 149 (3), 1190-1204. doi: http://doi.org/10.1210/en.2007-0665

[3] Clement, P. B. (2007). The pathology of endometriosis - A survey of the many faces of a common disease emphasizing diagnostic pitfalls and unusual and newly appreciated aspects. Advances in Anatomic Pathology, 14 (4), 241-260. doi: http:// doi.org/10.1097/pap.0b013e3180ca7d7b

[4] Prokhorova, M. V., Manukhin, I. B., Gevorkyan, M. A., Smirnova, S. O. (2013). Suppressive therapy for chronic pelvic pain in patients with external genital endometriosis. Obstetrics and gynecology, 9, 101-104.

[5] Bourdon, M., Santulli, P., Chouzenoux, S., Maignien, C., Bailly, K., Andrieu, M. et. al. (2018). The Disease Phenotype of Adenomyosis-Affected Women Correlates With Specific Serum Cytokine Profiles. Reproductive Sciences, 26 (2), $198-206$. doi: http://doi.org/10.1177/1933719118816852

[6] Ozcelik, K., Capar, M., Ucar, M. G., Cakur, T., Ozcelik, F., Ilhan, T. T. (2016). Are cytokine levels in serum, endometrial tissue, and peritoneal fluid a promising predictor to diagnosis of endometriosis-adenomyosis? Clinical and Experimental Obstetrics \& Gynecology, 43 (4), 569-572. 
[7] Wang, X.-Q., Zhou, W.-J., Luo, X.-Z., Tao, Y., Li, D.-J. (2017). Synergistic effect of regulatory T cells and proinflammatory cytokines in angiogenesis in the endometriotic milieu. Human Reproduction, 32 (6), 1304-1317. doi: http://doi.org/10.1093/ humrep/dex067

[8] Zaynetdinova, L. F., Telesheva, L. F., Koryaushkina, A. V. (2020). Features of local immunity in women with endometriosis and genital infection. Russian Journal of Infection and Immunity, 10 (1), 145-158. doi: http://doi.org/10.15789/ 2220-7619-fol-1192

[9] Nowak, N. M., Fischer, O. M., Gust, T. C., Fuhrmann, U., Habenicht, U.-F., Schmidt, A. (2008). Intraperitoneal inflammation decreases endometriosis in a mouse model. Human Reproduction, 23 (11), 2466-2474. doi: http://doi.org/10.1093/ humrep/den189

[10] Koninckx, P. R., Ussia, A., Tahlak, M., Adamyan, L., Wattiez, A., Martin, D. C. et. al. (2019). Infection as a potential cofactor in the genetic-epigenetic pathophysiology of endometriosis: a systematic review. Facts Views and Vision in Obgyn, 11 (3), $209-216$.

[11] Glukhov, E. Yu., Oboskalova, T. A., Urakov, A. V. (2014). Possibilities of argon plasma coagulation in the combination therapy of severe endometriosis. Obstetrics and gynecology, 2, 53-56.

[12] Sugimoto, K., Nagata, C., Hayashi, H., Yanagida, S., Okamoto, A. (2015). Use of dienogest over 53 weeks for the treatment of endometriosis. Journal of Obstetrics and Gynaecology Research, 41 (12), 1921-1926. doi: http://doi.org/10.1111/jog.12811

[13] Frackiewicz, E. J., Zarotsky, V. (2003). Diagnosis and treatment of endometriosis. Expert Opinion on Pharmacotherapy, 4 (1), 67-82. doi: http://doi.org/10.1517/14656566.4.1.67

[14] Adamyan, L. V., Aznaurova, Y. B. (2015). Molecular aspects of endometriosis. Problemy Reproduktsii, 21 (2), 66-77. doi: http://doi.org/10.17116/repro201521266-77

[15] Adamian, L. V., Kulakov, V. I., Andreeva, E. N. (2006). Endometriosis. Moscow: Medicine, 411.

[16] Alkatout, I., Wedel, T., Maass, N. (2018). Combined treatment of endometriosis: radical yet gentle. Aktuelle Urologie, 49 (1), 60-72. doi: http://doi.org/10.1055/s-0043-122175

[17] Soares, S. R., Martínez-Varea, A., Hidalgo-Mora, J. J., Pellicer, A. (2012). Pharmacologic therapies in endometriosis: a systematic review. Fertility and Sterility, 98 (3), 529-555. doi: http://doi.org/10.1016/j.fertnstert.2012.07.1120

[18] Lykov, A. P., Sakhno, L. V., Kozlov, V. A. (1998). Production of cytokines (interleukins $1 \beta$ and 2, tumor necrosis factor $\alpha$ by blood mononuclear cells in the Chernobyl accident liquidators. Immunology, 4, 56-57.

[19] Glants, S. M. (1999). Biomedical statistics. Moscow: Praktika, 459.

[20] Xie, Q., He, H., Wu, Y., Zou, L., She, X., Xia, X., Wu, X. (2019). Eutopic endometrium from patients with endometriosis modulates the expression of CD36 and SIRP- $\alpha$ in peritoneal macrophages. Journal of Obstetrics and Gynaecology Research, 45 (5), 1045-1057. doi: http://doi.org/10.1111/jog.13938

[21] Sukhikh, G. T., Sotnikova, N. Y., Antsiferova, Y. S., Posiseeva, L. V., Veryasov, V. N., Vanko, L. V. (2004). Cytokine Production by Immunocompetent Cells of Peritoneal Fluid in Women with External Genital Endometriosis. Bulletin of Experimental Biology and Medicine, 137 (6), 568-571. doi: http://doi.org/10.1023/b:bebm.0000042714.32571.6b

[22] Kodati, V. L., Govindan, S., Movva, S., Ponnala, S., Hasan, Q. (2008). Role of shigella infection in endometriosis: A novel hypothesis. Medical Hypotheses, 70 (2), 239-243. doi: http://doi.org/10.1016/j.mehy.2007.06.012

[23] Khan, K. N., Fujishita, A., Hiraki, K., Kitajima, M., Nakashima, M., Fushiki, S., Kitawaki, J. (2018). Bacterial contamination hypothesis: a new concept in endometriosis. Reproductive Medicine and Biology, 17 (2), 125-133. doi: http://doi.org/10.1002/ $\mathrm{rmb} 2.12083$

[24] Tai, F.-W., Chang, C., Chiang, J.-H., Lin, W.-C., Wan, L. (2018). Association of Pelvic Inflammatory Disease with Risk of Endometriosis: A Nationwide Cohort Study Involving 141,460 Individuals. Journal of Clinical Medicine, 7 (11), 379. doi: http://doi.org/10.3390/jcm7110379

[25] Chadchan, S. B., Cheng, M., Parnell, L. A., Yin, Y., Schriefer, A., Mysorekar, I. U., Kommagani, R. (2019). Antibiotic therapy with metronidazole reduces endometriosis disease progression in mice: A potential role for gut microbiota. Human Reproduction, 34, 1106-1116. doi: http://doi.org/10.1093/humrep/dez041

Received date 16.11.2020

(C) The Author(s) 2021

Accepted date 11.02.2021

Published date 25.05.2021

This is an open access article under the Creative Commons CC BY license

How to cite: Shcherbina, N., Chekhunova, A. (2021). Treatment optimization of patients with genital endometriosis. EUREKA: Health Sciences, 3, 3-8. doi: http://doi.org/10.21303/2504-5679.2021.001682 